\title{
Estudio de la viabilidad técnica, economica y financiera de la instalación de alfalfa (Medicago sativa) en la Costa Norte del Perú
}

\author{
Study of the technical, economic and financial viability of the estableshment of alfalfa (Medicago \\ sativa) in the North Coast of Peru
}

Edgardo A. Vilcara C. ${ }^{1}$; Fernando J. Passoni T. ${ }^{2}$

\section{Resumen}

El presente estudio fue realizado con la finalidad de determinar la viabilidad económica, técnica y financiera de la instalación de alfalfa (Medicago sativa), variedad Suprema, para su venta como forraje verde a las empresas dedicadas a la crianza de ganado vacuno. El proyecto, con un tamaño de 30 hectáreas, fue desarrollado en el distrito de Paiján, provincia de Ascope, departamento de La Libertad, gracias a la cercanía del mercado objetivo, su fácil accesibilidad y a las posibilidades de expansión de la empresa. El presente estudio eligió el cultivo de alfalfa variedad Suprema por sus bondades forrajeras; además, a nivel del mercado objetivo, permitirá cubrir el $8 \%$ de la demanda global de alfalfa. El análisis técnico indicó que el proyecto de alfalfa es factible, por la sencillez y flexibilidad de la tecnología que permite establecer y manejar adecuadamente el cultivo. El análisis económico y financiero del proyecto determinó su viabilidad mostrando indicadores de rentabilidad muy significativos.

Palabras clave: alfalfa; análisis económico y financiero; proyecto; viabilidad.

\begin{abstract}
This study was carried out to determine the technical, economic and financial viability of the establishment of the alfalfa crop (Medicago sativa var. Suprema) to be sold as green fodder to cattle enterprises. The project area will have a size of 30 hectares and will be located in the District of Paiján, Ascope Province, Department of La Libertad. The location is primarily due to the proximity of the target market, easy accessibility and potential for expansion. The Suprema alfalfa variety was chosen because of its excellent attributes as a forage crop. The participation of the present project, at the target market level, will cover $8 \%$ of the global demand for alfalfa. The technical analysis showed that the project is feasible because of the simplicity and flexibility of the technology that allows to establish and to manage the crop. The economic and financial analysis of the project established its viability showing significant profitability indicators.
\end{abstract}

Key words: alfalfa; economic and financial analysis; project; viability.

\section{Introducción}

En la costa norte del Perú, se viene desarrollando desde el 2006 "El Plan Ganadero de la Región La Libertad" el cual durará hasta el 2015. Este plan tiene entre sus objetivos organizar a los productores agrarios y mejorar e incrementar la actividad ganadera del departamento en mención, involucrando a los gobiernos locales, regionales y nacionales, tanto es así que un moderno centro de producción agrícola ha sido instalado en el distrito de Huanchaco, con vacas lecheras de la raza Jersey y Holstein, con el objetivo de mejorar los productos lácteos para competir en el mercado exterior.

Diversas actividades se vienen realizando y en ellas se evidencia un claro interés en el sector ganadero, mayormente vacuno, el cual está creciendo año tras año. Esta situación plantea la necesidad de producir más forraje para satisfacer la creciente demanda; es así que el cultivo de alfalfa (Medicago sativa) se presenta como una buena alternativa para su producción. Según Del Pozo (1984), la alfalfa pertenece a la familia de las leguminosas y su nombre científico es Medicago sativa; es una planta perenne, vivaz y de porte erecto, con una raíz pivotante que, conjuntamente con la corona (zona meristemática), permite tomar decisiones sobre el manejo.

La alfalfa variedad Suprema es considerada adecuada para el cultivo, en el norte del Perú, por sus bondades forrajeras. Se puede sembrar todo el año, siempre y cuando tenga riego, y es capaz de soportar hasta 10 cortes por hectárea por año con un rendimiento de hasta $10 \mathrm{TM}$ de forraje verde por corte (Carlessi SAC, 2008). Según Narrea (2009) las principales plagas que atacan a la alfalfa en el norte del Perú son: Epinotia aporema, Prodiplosis longifila, Aphis craccivora, Therioaphis trifolii, Spodoptera eridania y Spodoptera frugiperda. Sin embargo, se espera que la variedad Suprema muestre buena resistencia.

La preparación adecuada del terreno es determinante para lograr altos rendimientos del cultivo durante varios años. Las labores se deberán efectuar con la anticipación suficiente como para permitir la acumulación de agua en el perfil del suelo (Flórez et al., 1992).

La evaluación de proyectos no tiene teoría propia y cerrada, sino más bien se hace combinando diversas disciplinas

1 Bachiller en Ciencias, Facultad de Agronomía. Universidad Nacional Agraria La Molina, Lima, Perú. E-mail: edcardens@gmail.com

2 Profesor Asociado del Departamento de Fitotecnia. Universidad Nacional Agraria La Molina, Lima, Perú. E-mail: fpassoni@lamolina.edu.pe 
como la economía, finanzas, planeamiento estratégico e ingeniería (Kafka, 1993). Según Torres (1999), el proceso de evaluación se realiza para saber si a lo largo de la vida útil del proyecto los beneficios superan a los costos.

El punto de equilibrio económico es aquel en el cual los ingresos totales y los gastos son iguales; es decir, no existe utilidad ni pérdida (Kaye, 1998). Adicionalmente, Torres (1992) señala que los indicadores económicos y financieros son el Valor Actual Neto (VAN), la Tasa Interna de Retorno (TIR), la relación Beneficio-Costo $(\mathrm{B} / \mathrm{C})$ y el Periodo de Recuperación de la Inversión (PRI).

Para Sapag y Sapag (1995) el Valor Actual Neto (VAN) representa el excedente generado por un proyecto, en términos absolutos, después de haber cubierto los costos de inversión, operación y uso de capital. Estos mismos autores definen a la Tasa Interna de Retorno (TIR) de un proyecto de inversiones como la tasa de descuento que iguala el valor actual de los costos previstos. Es aquella tasa de descuento para la cual el VAN resulta igual a cero. Otro criterio de evaluación es el periodo de recuperación de la inversión, mediante el cual se puede determinar el número de periodos necesarios para poder recuperar la inversión inicial (Chu, 2003).

El presente estudio tiene por objetivo general evaluar la viabilidad técnica, económica y financiera de la instalación y producción de alfalfa (Medicago sativa) var. Suprema bajo las condiciones imperantes en la costa norte del Perú complementando esta tarea con la identificación del mercado y análisis de su tamaño, preferencias y evidencias de consumo, así como con la determinación de la inversión total del proyecto y su rentabilidad.

\section{Materiales y métodos Hipótesis}

El establecimiento, producción y comercialización de la alfalfa en verde es técnica, económica y financieramente viable de realizarse bajo las condiciones de la costa norte del Perú.

\section{Obtención y procesamiento de información}

Se recurrió a la obtención de información de fuentes primarias y secundarias. Entre las fuentes primarias, se realizaron entrevistas con especialistas de la región de $\mathrm{La}$ Libertad (universidades y Ministerio de Agricultura) sobre técnicas de cultivo, tecnología y manejo integrado de plagas. Asimismo, se tomó contacto, por correo electrónico, con empresas semilleristas, agrícolas, ganaderas así como con productores y comercializadores de alfalfa. Las fuentes secundarias lo constituyen diversos libros, revistas $\mathrm{y}$ boletines que fueron revisados atentamente.

A partir de la información anterior, se realizaron los siguientes pasos:

1. Determinación de la localización del proyecto.

2. Estudio del mercado objetivo.

3. Determinación de la demanda de alfalfa.

4. Determinación de la oferta.

5. Instalación del cultivo.

6. Determinación de la inversión total del proyecto y el presupuesto de ingresos y egresos.

7. Evaluación económicofinanciera.

\section{Cultivo de alfalfa}

La preparación del terreno se hizo con maquinaria agrícola. Se incorporó materia orgánica y se emplearon fertilizantes orgánicos tanto en la siembra como durante la producción. La semilla fue de alfalfa (Medicago sativa) variedad Suprema y se inoculó con Rhizobium meliloti.

La siembra fue a mano y escalonada abarcando dos hectáreas por día hasta completar las 30 hectáreas. El riego fue por gravedad y espaciado en quincenas. Lo anterior permitió determinar a cuánto asciendería la instalación de una hectárea de alfalfa. Durante la producción se esperaron frecuencias de 8 a 10 cortes por hectárea por año.

\section{Indicadores económicos y financieros}

Se elaboró el estado de pérdidas y ganancias para determinar el impuesto a la renta, la utilidad neta del proyecto y el flujo de caja, tanto económico como financiero, para determinar los indicadores de rentabilidad. El Valor Actual Neto (VAN) se calculó al momento cero y representa el excedente generado por un proyecto, en términos absolutos, después de haber cubierto los costos de inversión, operación y de uso del capital.

La expresión del VAN económico fue el siguiente:

$\mathrm{VANE}=-\mathrm{IT}+\mathrm{FE} * \mathrm{FSA}$

donde:

VANE $=$ Valor actual neto económico

IT $=$ Inversión total del proyecto

$\mathrm{FE}=$ Flujo económico

$\mathrm{FSA}=$ Factor simple de actualización

La expresión del VAN financiero fue el siguiente:

$\mathrm{VANF}=-\mathrm{AP}+\mathrm{FF} * \mathrm{FSA}$

donde:

$$
\begin{aligned}
& \text { VANF = Valor actual neto financiero } \\
& \mathrm{AP}=\text { Aporte propio de la empresa } \\
& \mathrm{FF}=\text { Flujo financiero } \\
& \text { FSA = Factor simple de actualización }
\end{aligned}
$$

Los criterios de evaluación fueron:

VAN $>0$ Se acepta el proyecto

VAN $<0$ Se rechaza el proyecto

$\mathrm{VAN}=0 \quad \mathrm{El}$ proyecto es indiferente

La Tasa Interna de Retorno (TIR) es aquella tasa de oportunidad o tasa de requerimiento requerido (TRR) que hace el VAN exactamente igual a cero e indica la rentabilidad promedio anual que genera el capital que permanece invertido en el proyecto.

Para la evaluación económica, la TIRE tiene la siguiente expresión:

- IT + FE $*$ FSA $(r)=0$

donde $\mathrm{r}=\mathrm{TIRE}=$ Tasa interna de retorno económico

Para la evaluación financiera, la TIRF tiene la siguiente expresión:

$-\mathrm{AP}+\mathrm{FF} * \mathrm{FSA}(\mathrm{r})=0$

donde $r=T I R F=$ Tasa interna de retorno financiero

Los criterios de evaluación son:

TIR > TRR Se acepta el proyecto

TIR $<$ TRR Se rechaza el proyecto

Relación Beneficio-Costo (B/C) es la cantidad de excedente generado por unidad de inversión después de haber cubierto los costos de operación y de capital. Matemáticamente la expresión es la siguiente: 


$$
\mathrm{B} / \mathrm{C}=\frac{\mathrm{FE}{ }^{*} \mathrm{FSA}}{\mathrm{IT}}
$$

donde:

$$
\begin{aligned}
& \mathrm{B} / \mathrm{C}=\text { Relación beneficio-costo } \\
& \mathrm{FE}=\text { Flujo económico } \\
& \mathrm{FSA}=\text { Factor simple de actualización } \\
& \mathrm{IT}=\text { Inversión total del proyecto }
\end{aligned}
$$

La regla de decisión es la siguiente: un proyecto debe aceptarse si la relación beneficio-costo es mayor a 1 .

El Periodo de Recuperación de la Inversión (PRI) es el número de periodos necesarios para que el inversionista logre recuperar la inversión inicial. La regla de decisión es: la recuperación de la inversión inicial debe ocurrir dentro del horizonte del proyecto para aceptarse. Matemáticamente, se calcula por la suma acumulada del número de periodos hasta alcanzar el monto de la inversión, así:

\section{Resultados y discusión}

\section{Tamaño y localización}

El proyecto tuvo un tamaño de 30 hectáreas. El método del ranking de factores determinó que el presente proyecto fuera desarrollado en el distrito de Paiján, provincia de Ascope, departamento de La Libertad.

\section{Estudio de mercado}

El mercado objetivo fueron los distritos de Casa Grande, Chicama y Paiján.

\section{Características de la demanda del mercado objetivo}

La demanda de forraje (consumo) según la cantidad de ganado vacuno lechero existente en los distritos en estudio

$\begin{array}{lllll}\text { Periodos (P) } & 0 & 1 & 2 & 3 \\ \text { Flujo de Caja } & \begin{array}{l}\text { Inversión } \\ \text { Inicial = 50 }\end{array} & 10(\mathrm{a}) & 20(\mathrm{~b}) & 30(\mathrm{c}) \\ \text { P acumulados } & & 10(\mathrm{a}) & 30(\mathrm{a}+\mathrm{b}) & 60(\mathrm{a}+\mathrm{b})+\mathrm{c}\end{array}$

PRI Entre 2 y 3 se muestra en la Tabla 1. En este se aprecia la relación directa que existe entre el número de cabezas de ganado vacuno y la demanda de forraje, la cual va en aumento constante.

\section{Proyección de la demanda}

El número de cabezas de ganado vacuno lechero fue proyectado por la ecuación polinomial $\mathrm{Y}=23,69 \mathrm{X}+$ $6051,6\left(\mathrm{R}^{2}=0,9681\right)$. Con un promedio de 500 kilos de peso vivo por animal, el consumo de materia seca (MS) fue de $3 \mathrm{~kg}$ por vacuno por día o su equivalente a 15 kilos de alfalfa verde (Tabla 2).

El incremento del ganado vacuno conlleva el aumento de consumo de forrajes. El proyecto ingresará al mercado de forrajes proveyendo un producto de calidad y supliéndolo de manera constante en los próximos ocho años, a partir del 2009 en adelante.

\section{Participación del Proyecto}

En la Tabla 3 se puede apreciar la participación del proyecto a lo largo de los ocho años de su duración. El presente proyecto aporta un $8 \%$ de la materia seca requerida (MS). Se espera crecer a lo largo de los años a fin de sostener la demanda de alimento por parte del ganado vacuno presente en estos distritos.

\section{Instalación del cultivo de alfalfa}

La instalación de una hectárea de alfalfa equivale a $\mathrm{S} /$. $3.198,00$ y las 30 ha a S/. 105.840,00 lo cual incluye asistencia técnica y guardianía. Por cada hectárea de alfalfa se requerirán ocho jornales para la preparación del terreno; tres jornales para la siembra; once jornales para las labores culturales; seis horas de maquinaria agrícola y otros, tal como se señala en la Tabla 4.

\section{Inversiones y financiamiento}

La inversión total es el requerimiento necesario para la puesta en marcha del proyecto y comprende la inversión fija (activos fijos e intangibles) y el capital de trabajo. La

Tabla 1. Demanda global del mercado objetivo (Casa Grande, Chicama y Paiján del 2000 al 2007)

\begin{tabular}{ccc}
\hline Año & $\mathbf{N}^{\circ}$ de cabezas vacuno lechero & Consumo kg MS/año \\
\hline 2000 & 6071 & 6647667 \\
2001 & 6092 & 6671275 \\
2002 & 6128 & 6709880 \\
2003 & 6143 & 6726486 \\
2004 & 6178 & 6764366 \\
2005 & 6210 & 6800288 \\
2006 & 6221 & 6812173 \\
2007 & 6223 & 6814561 \\
\hline
\end{tabular}

Fuente: MINAG, 2008

Tabla 2. Demanda proyectada de materia seca (MS) por ganado vacuno en Casa Grande, Chicama y Paiján

\begin{tabular}{lrc}
\hline Año & $\mathbf{N}^{\circ}$ de cabezas vacuno & Consumo MS(kg/año) \\
\hline 2009 & 6289 & 6886190 \\
2010 & 6312 & 6912164 \\
2011 & 6336 & 6938139 \\
2012 & 6360 & 6964113 \\
2013 & 6384 & 6990088 \\
2014 & 6407 & 7016062 \\
2015 & 6431 & 7042036 \\
2016 & 6455 & 7068011 \\
\hline
\end{tabular}

Fuente: Elaboración propia. 
Tabla 3. Participación del proyecto en el mercado objetivo (Casa Grande, Chicama y Paiján)

\begin{tabular}{cccccc}
\hline Año & $\begin{array}{c}\text { Consumo } \\
\text { MS/año } \\
(\mathbf{k g})\end{array}$ & $\begin{array}{c}\text { Oferta MS/año } \\
(\mathbf{k g})\end{array}$ & $\begin{array}{c}\text { Demanda } \\
\text { Insatisfecha } \\
\text { (kg MS) }\end{array}$ & $\begin{array}{c}\text { Aporte del } \\
\text { Proyecto } \\
\text { ( kg MS) }\end{array}$ & $\begin{array}{c}\text { Demanda por } \\
\text { satisfacer } \\
\text { (kg MS) }\end{array}$ \\
\hline 2009 & 6886190 & 4010000 & 2876190 & 390000 & 2486190 \\
2010 & 6912164 & 4223580 & 2688584 & 480000 & 2208584 \\
2011 & 6938139 & 4437160 & 2500979 & 600000 & 1900979 \\
2012 & 6964113 & 4650740 & 2313373 & 600000 & 1713373 \\
2013 & 6990088 & 4864320 & 2125768 & 600000 & 1525768 \\
2014 & 7016062 & 5077900 & 1938162 & 600000 & 1338162 \\
2015 & 7042036 & 5291480 & 1750556 & 600000 & 1150556 \\
2016 & 7068011 & 5505060 & 1562951 & 600000 & 962951 \\
\hline
\end{tabular}

inversión total del proyecto asciende a S/. 535.578,00 tal como se muestra en la Tabla 5.

Los activos fijos ascendieron a S/. 298.633,00 y los intangibles a S/. 112.190,00 que comprendieron los gastos de estudio (S/. 3000,00); gastos de constitución y organización (S/. 650,00) así como los gastos de instalación del cultivo de alfalfa (Medicago sativa) variedad Suprema (S/. 105.840,00). El capital de trabajo inicial fue

Tabla 4. Gastos de instalación de alfalfa (medicago sativa)

\begin{tabular}{|c|c|c|c|c|}
\hline ACTIVIDAD & Unidad de medida & $\begin{array}{l}\text { Cantidad } \\
\text { Utilizada }\end{array}$ & $\begin{array}{c}\text { Costo unitario } \\
\text { (S/.) }\end{array}$ & Costo Total (S/.) \\
\hline \multicolumn{5}{|l|}{ MANO DE OBRA } \\
\hline \multicolumn{5}{|l|}{ Preparación del terreno } \\
\hline Pica, despaje y quema & Jornal & 2 & 17 & 34 \\
\hline Limpieza de canales y acequias & Jornal & 2 & 17 & 34 \\
\hline Confección de melgas & Jornal & 2 & 17 & 34 \\
\hline Tomeo y riego de machaco & Jornal & 2 & 17 & 34 \\
\hline \multicolumn{5}{|l|}{ Siembra } \\
\hline Desinfección y siembra & Jornal & 2 & 17 & 34 \\
\hline Resiembra a mano & Jornal & 1 & 17 & 17 \\
\hline \multicolumn{5}{|l|}{ Labores culturales } \\
\hline Abonamiento & Jornal & 2 & 17 & 34 \\
\hline Deshierbo & Jornal & 2 & 17 & 34 \\
\hline Despique & Jornal & 2 & 17 & 34 \\
\hline Riegos & Jornal & 3 & 17 & 51 \\
\hline \multicolumn{5}{|l|}{ MAQUINARIA AGRICOLA } \\
\hline Aradura & Hora/máquina & 2 & 70 & 140 \\
\hline Rastrillo y Gradeo & Hora/máquina & 2 & 70 & 140 \\
\hline Nivelación & Hora/máquina & 2 & 70 & 140 \\
\hline \multicolumn{5}{|l|}{ INSUMOS } \\
\hline Semilla & $\mathrm{kg}$ & 25 & 35 & 875 \\
\hline Materia orgánica & ton & 5 & 12,5 & 62,5 \\
\hline \multicolumn{5}{|l|}{ Fertilizantes } \\
\hline Sulfato de potasio & $\operatorname{sacos}$ & 3,2 & 101 & 323,2 \\
\hline Superfosfato triple de calcio & $\operatorname{sacos}$ & 7 & 122 & 854 \\
\hline Agua & $\mathrm{m}^{3}$ & 6000 & 0,045 & 270 \\
\hline Fletes & $\mathrm{kg}$ & 533 & 0,1 & 53,3 \\
\hline Costo ha & & & & 3198 \\
\hline Costo por 30 ha & & & & 95940 \\
\hline Asistencia técnica & & & & 7500 \\
\hline Guardianía x 3 meses & & & & 2400 \\
\hline COSTO TOTAL DE INSTALACION & & & & 105840 \\
\hline
\end{tabular}


equivalente al $80 \%$ de los costos de producción y ascendió a S/. 124.755,60. El financiamiento del proyecto estuvo a cargo de COFIDE quien dio un crédito pagadero en cinco años, a una tasa del $14 \%$.

La estructura del financiamiento está referida a la procedencia de los recursos para las inversiones iniciales. Como se aprecia en la Tabla 6, se hizo un préstamo de S/. $349.954(65,34 \%)$ y la empresa aportará S/. $185.625(34,66$ $\%$ ) para completar los S/. 535.579 que corresponden a la inversión total del proyecto.

Pago de intereses y amortización del préstamo

El préstamo se pagará con una tasa del $14 \%$ de interés al rebatir. Los pagos de intereses y amortizaciones serán anuales y se pagará en amortizaciones constantes durante cinco años (Tabla 7).

Costo de capital promedio ponderado

Es el promedio de los costos financieros de los préstamos a largo plazo más el costo de oportunidad del capital contable de la compañía, debido a que se están utilizando ambos recursos (Chu, 2003). El costo capital promedio ponderado para el presente proyecto resultó ser de $13,34 \%$ (Tabla 8 ).

Tabla 5. Inversión total del proyecto

\begin{tabular}{lc}
\hline INVERSIÓN TOTAL (S/.) & $\mathbf{5 3 5 . 5 7 8 , 6 0}$ \\
\hline INVERSIÓN FIJA & $410.823,00$ \\
Activo Fijo & $298.633,00$ \\
Activos Intangibles & $112.190,00$ \\
CAPITAL DE TRABAJO & $124.755,60$ \\
\hline Fuente: Elaboración propia.
\end{tabular}

\section{Presupuesto de ingresos y egresos}

\section{Ingresos}

Los ingresos estuvieron dados por la venta de alfalfa en verde (FV) a S/. 0,20 el kilo. Se estimaron ingresos de S/. 390.000 el primer año (producción al $65 \%$ ); 480.000 el segundo año (producción al $80 \%$ ) y de S/. 600.000 (producción al $100 \%$ ) desde el tercero hasta el octavo año. En la Tabla 9 se presenta la producción e ingresos por venta de alfalfa para los ocho años del proyecto.

\section{Egresos}

Están dados por los costos de producción (S/. 155.945,00 anuales); gastos administrativos y de ventas (S/. 89.910 anuales) y los gastos financieros, que son los intereses del préstamo, los mismos que se muestran en las Tablas 10, 11 y 12 .

\section{Estados económico financieros}

En la Tabla 13 se muestra el Estado de Pérdidas y Ganancias del proyecto. Se aprecia que la utilidad neta es positiva a lo largo del horizonte del proyecto. En la Tabla 14 se muestra el Flujo de Caja Económico y Financiero; el económico considera a los inversionistas como única fuente de financiamiento mientras que el financiero considera la existencia de dos fuentes, que son el préstamo y el aporte propio. Con los flujos de caja se realizó la evaluación del proyecto.

\section{Evaluación Económica y Financiera}

Para el cálculo del VANE y del VANF se consideró una Tasa de Retorno Requerida (TRR) de $20 \%$, dando como resultado un VANE de S/. 333.091 por lo cual el proyecto debe ser aceptado al ser mayor que cero, tal como lo mencionan Beltrán y Cueva (2000). Con la TRR

Tabla 6. Estructura del financiamiento

\begin{tabular}{lcccccccc}
\hline & \multirow{2}{*}{ ACTIVO FIJO } & \multicolumn{2}{c}{$\begin{array}{c}\text { ACTIVOS } \\
\text { INTANGIBLES }\end{array}$} & \multicolumn{2}{c}{$\begin{array}{c}\text { CAPITAL DE } \\
\text { TRABAJO }\end{array}$} & \multicolumn{2}{c}{$\begin{array}{c}\text { INVERSIÓN } \\
\text { TOTAL (S/.) }\end{array}$} \\
\hline \multirow{3}{*}{ Préstamo } & $\mathrm{S} /$. & $\%$ & $\mathrm{~S} /$. & $\%$ & $\mathrm{~S} /$. & $\%$ & TOTAL & $\%$ \\
Aporte Propio & 209.043 & 70 & 78.533 & 70 & $62.377,8$ & 50 & 349.954 & 65,34 \\
& 89.590 & 30 & 33.657 & 30 & $62.377,8$ & 50 & 185.625 & 34,66 \\
& $\mathbf{2 9 8 . 6 3 3}$ & 100 & $\mathbf{1 1 2 . 1 9 0}$ & 100 & $\mathbf{1 2 4 . 7 5 5 , 6}$ & 100 & $\mathbf{5 3 5 . 5 7 9}$ & $\mathbf{1 0 0}$ \\
\hline
\end{tabular}

Fuente: Elaboración propia.

Tabla 7. Programa de pago de la deuda

\begin{tabular}{|c|c|c|c|c|c|c|c|}
\hline & & 0 & 1 & 2 & 3 & 4 & 5 \\
\hline Préstamo & 349954 & & & & & & \\
\hline Cuotas & 5 & & & & & & \\
\hline Tasa interés & $14 \%$ & & & & & & \\
\hline Saldo deudor & & 349954 & 279963 & 209973 & 139982 & 69991 & 0 \\
\hline Amortización & & & 69991 & 69991 & 69991 & 69991 & 69991 \\
\hline Intereses & & & 48994 & 39195 & 29396 & 19597 & 9799 \\
\hline
\end{tabular}

Tabla 8. Costo de capital promedio ponderado

\begin{tabular}{|c|c|c|c|c|c|c|}
\hline \multirow[t]{2}{*}{ FUENTES } & \multicolumn{2}{|c|}{ INVERSIONES } & \multirow{2}{*}{$\begin{array}{c}\text { COSTO } \\
\text { INTERÉS } \\
(\%)\end{array}$} & \multirow{2}{*}{$\begin{array}{c}\text { ESCUDO } \\
\text { FISCAL } \\
(\%)\end{array}$} & \multirow{2}{*}{$\begin{array}{c}\text { COSTO } \\
\text { NETO } \\
(\%)\end{array}$} & \multirow{2}{*}{$\begin{array}{c}\text { CCPP } \\
(\%) \\
\end{array}$} \\
\hline & $(\mathrm{S} /)$. & $(\%)$ & & & & \\
\hline Banca & 349954 & 65,34 & 14 & 4,2 & 9,8 & 6,40 \\
\hline Aporte Propio & 185625 & 34,66 & 20 & 0 & 20 & 6,93 \\
\hline Total & 535579 & 100 & & & & 13,34 \\
\hline
\end{tabular}

Fuente: Elaboración propia. 
Tabla 9. Producción y venta de alfalfa (Medicago sativa)

\begin{tabular}{cccc}
\hline Año & Productividad $(\%)$ & $\begin{array}{c}\text { Rendimiento FV } \\
(\mathrm{kg} / 30 \text { has })\end{array}$ & $\begin{array}{c}\text { Venta Total } \\
(\mathrm{S} / .)\end{array}$ \\
\hline 1 & 65 & 1950000 & 390000 \\
2 & 80 & 2400000 & 480000 \\
3 & 100 & 3000000 & 600000 \\
4 & 100 & 3000000 & 600000 \\
5 & 100 & 3000000 & 600000 \\
6 & 100 & 3000000 & 600000 \\
7 & 100 & 3000000 & 600000 \\
8 & 100 & 3000000 & 600000 \\
\hline
\end{tabular}

Tabla 10. Costos de producción

\begin{tabular}{lcccccccc}
\hline & 1 & 2 & 3 & 4 & 5 & 6 & 7 & 8 \\
\hline Insumos & 36828 & 36828 & 36828 & 36828 & 36828 & 36828 & 36828 & 36828 \\
Mano Obra & 106133 & 106133 & 106133 & 106133 & 106133 & 106133 & 106133 & 106133 \\
Costos Indir. & 12984 & 12984 & 12984 & 12984 & 12984 & 12984 & 12984 & 12984 \\
$\quad$ TOTAL & 155945 & 155945 & 155945 & 155945 & 155945 & 155945 & 155945 & 155945 \\
\hline
\end{tabular}

Tabla 11. Gastos administrativos y de ventas

\begin{tabular}{lllllllll}
\hline & 1 & 2 & 3 & 4 & 5 & 6 & 7 & 8 \\
\hline Administrador & 38295 & 38295 & 38295 & 38295 & 38295 & 38295 & 38295 & 38295 \\
Asistente contable & 19980 & 19980 & 19980 & 19980 & 19980 & 19980 & 19980 & 19980 \\
Seguridad & 13320 & 13320 & 13320 & 13320 & 13320 & 13320 & 13320 & 13320 \\
Secretaria & 18315 & 18315 & 18315 & 18315 & 18315 & 18315 & 18315 & 18315 \\
Total & 89910 & 89910 & 89910 & 89910 & 89910 & 89910 & 89910 & 89910 \\
\hline
\end{tabular}

Tabla 12. Gastos financieros

\begin{tabular}{|l|c|c|c|c|c|c|c|c|}
\hline Año & 1 & 2 & 3 & 4 & 5 & 6 & 7 & 8 \\
\hline Gastos Financieros & 48994 & 39195 & 29396 & 19597 & 9799 & 0 & 0 & 0 \\
\hline
\end{tabular}

Tabla 13. Estado de pérdidas y ganancias

\begin{tabular}{|l|r|r|r|r|r|r|r|r|}
\hline AÑOS & 1 & 2 & 3 & 4 & 5 & 6 & 7 & 8 \\
\hline Ventas & 390000 & 480000 & 600000 & 600000 & 600000 & 600000 & 600000 & 600000 \\
\hline $\begin{array}{l}\text { Utilidad por venta } \\
\text { del terreno }\end{array}$ & 0 & 0 & 0 & 0 & 0 & 0 & 0 & 21600 \\
\hline Costos & 182346,75 & 182346,754 & 182346,754 & 182346,754 & 182346,754 & 182346,754 & 182346,754 & 182346,754 \\
\hline -Producción & 155944,5 & 155944,5 & 155944,5 & 155944,5 & 155944,5 & 155944,5 & 155944,5 & 155944,5 \\
\hline -Depreciación & 12378,50 & 12378,50 & 12378,50 & 12378,50 & 12378,50 & 12378,50 & 12378,50 & 12378,50 \\
\hline -Amortización & 14023,75 & 14023,75 & 14023,75 & 14023,75 & 14023,75 & 14023,75 & 14023,75 & 14023,75 \\
Activos Intangibles & & & & & 4175 & \\
\hline Utilidad Bruta & 207653,25 & 297653,247 & 417653,247 & 417653,247 & 417653,247 & 417653,247 & 417653,247 & 439253,247 \\
\hline Gastos & 138904 & 129105 & 119306 & 109507 & 99709 & 89910 & 89910 & 89910 \\
\hline -Administrativos & 89910 & 89910 & 89910 & 89910 & 89910 & 89910 & 89910 & 89910 \\
\hline -Financieros & 48994 & 39195 & 29396 & 19597 & 9799 & 0 & 0 & 0 \\
\hline Utilidad Imponible & 68750 & 168548 & 298347 & 308146 & 317944 & 327743 & 327743 & 349343 \\
\hline Impuestos (30\%) & 20625 & 50564 & 89504 & 92444 & 95383 & 98323 & 98323 & 104803 \\
\hline Utilidad Neta & 48125 & 117984 & 208843 & 215702 & 222561 & 229420 & 229420 & 244540 \\
\hline Impuesto Económico & 35323 & 62323 & 98323 & 98323 & 98323 & 98323 & 98323 & 104803 \\
\hline Impuesto Económico & 35323 & 62323 & 98323 & 98323 & 98323 & 98323 & 98323 & 104803 \\
\hline Impuesto Financiero & 20625 & 50564 & 89504 & 92444 & 95383 & 98323 & 98323 & 104803 \\
\hline Escudo Fiscal & 14698 & 11759 & 8819 & 5879 & 2940 & 0 & 0 & 0 \\
\hline
\end{tabular}


Tabla 14. Flujo de caja económico y financiero

\begin{tabular}{|c|c|c|c|c|c|c|c|c|c|}
\hline AÑOS & 0 & 1 & 2 & 3 & 4 & 5 & 6 & 7 & 8 \\
\hline \multicolumn{10}{|l|}{ I. INGRESOS } \\
\hline Ventas & 0 & 390000 & 480000 & 600000 & 600000 & 600000 & 600000 & 600000 & 600000 \\
\hline $\begin{array}{l}\text { Valor Recupero } \\
\text { Activo Fijo }\end{array}$ & 0 & 0 & 0 & 0 & 0 & 0 & 0 & 0 & 188210 \\
\hline $\begin{array}{l}\text { Valor Recupero } \\
\text { Capital Trabajo }\end{array}$ & 0 & 0 & 0 & 0 & 0 & 0 & 0 & 0 & 124756 \\
\hline TOTAL INGRESOS & 0 & 390000 & 480000 & 600000 & 600000 & 600000 & 600000 & 600000 & 912966 \\
\hline \multicolumn{10}{|l|}{ II. EGRESOS } \\
\hline Costos de Producción & 0 & 155944,5 & 155944,5 & 155944,5 & 155944,5 & 155944,5 & 155944,5 & 155944,5 & 155944,5 \\
\hline Gastos Administrativos & 0 & 89910 & 89910 & 89910 & 89910 & 89910 & 89910 & 89910 & 89910 \\
\hline Impuestos & 0 & 35323 & 62323 & 98323 & 98323 & 98323 & 98323 & 98323 & 104803 \\
\hline \multicolumn{10}{|l|}{ Inversiones: } \\
\hline Activos Fijos & 298633 & 0 & 0 & 0 & 0 & 8478,96 & 0 & 0 & 0 \\
\hline Activos Intangibles & 112190 & 0 & 0 & 0 & 0 & 0 & 0 & 0 & 0 \\
\hline Capital de Trabajo & 124756 & 0 & 0 & 0 & 0 & 0 & 0 & 0 & 0 \\
\hline TOTAL EGRESOS & 535579 & 281177 & 308177 & 344177 & 344177 & 352656 & 344177,5 & 344177 & 350657 \\
\hline $\begin{array}{l}\text { FLUJO DE CAJA } \\
\text { ECONÓMICO }\end{array}$ & -535579 & 108823 & 171823 & 255823 & 255823 & 247344 & 255823 & 255823 & 562308 \\
\hline $\begin{array}{l}\text { FINANCIAMIENTO } \\
\text { NETO }\end{array}$ & 349954 & -104287 & -97428 & -90568 & -83709 & -76850 & 0 & 0 & 0 \\
\hline Préstamos & 349954 & & & & & & & & \\
\hline Amortización & & -69991 & -69991 & -69991 & -69991 & -69991 & 0 & 0 & 0 \\
\hline Interés & & -48994 & -39195 & -29396 & -19597 & -9799 & 0 & 0 & 0 \\
\hline Escudo Fiscal & & 14698 & 11759 & 8819 & 5879 & 2940 & 0 & 0 & 0 \\
\hline $\begin{array}{l}\text { FLUJO DE CAJA } \\
\text { FINANCIERO }\end{array}$ & -185625 & 4535 & 74395 & 165254 & 172114 & 170493 & 255823 & 255823 & 562308 \\
\hline
\end{tabular}

anterior se obtuvo un VANF de S/. 401.168, por lo cual se concluye que el proyecto de inversión se debe aceptar debido a que supera la inversión total en dicha cantidad. Respecto a la Tasa Interna de Retorno (TIR), el proyecto fue aceptado dado que la TIRE $(35,00 \%)$ y la TIRF $(38,25 \%)$ fueron mayores que la TRR $(20 \%)$ y el CCPP $(13,34 \%)$, respectivamente. Asimismo, se obtuvo una relación beneficio costo $(\mathrm{B} / \mathrm{C})$ de $\mathrm{S} / .1,19$ y un periodo de recuperación del capital $(\mathrm{PRI})$ de tres años.

\section{Conclusiones}

La participación del presente proyecto, a nivel del mercado objetivo (Paiján, Casa Grande y Chicama), es mínima, siendo del $8 \%$ de la demanda global de la alfalfa. La inversión inicial del proyecto asciende a S/. 535.579. El análisis técnico indica que el proyecto es factible, por la sencillez y flexibilidad de la tecnología que permite establecer y manejar adecuadamente el cultivo. Asimismo, el proyecto presenta viabilidad económica y financiera tal como lo muestran los indicadores económicos y financieros que sustentan la rentabilidad del proyecto con una tasa de rendimiento requerida o costo de oportunidad de $20 \%$ y un costo de capital promedio ponderado de $13,34 \%$.

Se recomienda que el presente proyecto pase a un nivel de estudio de factibilidad con el fin de obtener una base que brinde un mayor sustento en la toma de decisiones.

\section{Literatura citada}

Beltrán, A. y Cueva, H. 2000. Ejercicios de evaluación privada de proyectos. (3ra. ed.). Lima, Perú: Centro de Investigación de la Universidad del Pacífico.

Carlessi, S.A.C. 2008. Características técnicas de la alfalfa variedad Suprema. Boletín Informativo. Lima, Perú.

Chu, M. 2003. Fundamentos de finanzas: Un enfoque peruano. (4ta. ed.) Lima, Perú:

Editorial Etiplus, S.R.L.

Del Pozo, M. 1984. La alfalfa, su cultivo y aprovechamiento. Madrid, España.

Flórez, A., Malpartida, E. y San Martin, F. (1992). Manual de forrajes para zonas áridas y semiáridas andinas. Red de Rumiantes Menores. Lima, Perú.

Kafka, F. 1993. Evaluación estratégica de proyectos de inversión. Lima, Perú: Editorial Biblioteca Universitaria. Universidad del Pacifico.

Kaye, A. 1998. Técnicas de los costos. Lima, Perú: Editores Asociados.

Ministerio de Agricultura [MINAG]. 2008. Estadística agraria mensual, Diciembre 2007. Lima, Perú: Dirección General de Investigación Agraria.

Narrea, M. 2009. Comunicación Personal. UNALM. Lima, Perú. 
Sapag, N. y Sapag, R. 1995. Preparación y evaluación de proyectos. (3ra. ed.). Colombia: Mc Graw-Hill.

Torres, L. 1992. Elementos para la formulación y evaluación de proyectos de inversión. Lima, Perú: Editorial UNALM.

Torres, L. 1999. Manual de formulación y evaluación de proyectos productivos. Lima, Perú: Editorial UNALM. 